\title{
Substructure Topology Preserving Simplification of Tetrahedral Meshes
}

\author{
Fabien Vivodtzev ${ }^{1}$, Georges-Pierre Bonneau ${ }^{2}$, Stefanie Hahmann ${ }^{2}$, and Hans Hagen ${ }^{3}$ \\ 1 CEA/CESTA (French Atomic Energy Commission) \\ 2 LJK, University of Grenoble and INRIA \\ 3 University of Kaiserslautern
}

\begin{abstract}
Interdisciplinary efforts in modeling and simulating phenomena have led to complex multi-physics models involving different physical properties and materials in the same system. Within a $3 \mathrm{~d}$ domain, substructures of lower dimensions appear at the interface between different materials. Correspondingly, an unstructured tetrahedral mesh used for such a simulation includes $2 \mathrm{~d}$ and $1 \mathrm{~d}$ substructures embedded in the vertices, edges and faces of the mesh.

The simplification of such tetrahedral meshes must preserve (1) the geometry and the topology of the $3 \mathrm{~d}$ domain, (2) the simulated data and (3) the geometry and topology of the embedded substructures. Although intensive research has been conducted on the first two goals, the third objective has received little attention. This paper focuses on the preservation of the topology of $1 \mathrm{~d}$ and $2 \mathrm{~d}$ substructures embedded in an unstructured tetrahedral mesh, during edge collapse simplification. We define these substructures as simplicial sub-complexes of the mesh, which is modeled as an extended simplicial complex. We derive a robust algorithm, based on combinatorial topology results, in order to determine if an edge can be collapsed without changing the topology of both the mesh and all embedded substructures. Based on this algorithm we have developed a system for simplifying scientific datasets defined on irregular tetrahedral meshes with substructures. The implementation of our system is discussed in detail. We demonstrate the power of our system with real world scientific datasets from electromagnetism simulations.
\end{abstract}

\section{Introduction}

In this paper we introduce a system that is able to robustly preserve surfaces and polylines defined as substructures in a tetrahedral mesh simplified by repeated edge collapses. This problem originates from an application in electromagnetism, as detailed in the next section. The surfaces (resp. polylines) we are dealing with consist in a subset of faces (resp. edges) of the tetrahedral mesh. Thus, collapsing an edge of the mesh may result in modification of the surfaces and polylines. Preserving these substructures during simplification is a new topic in the literature. There have several papers that have tackled a more specific issue: the preservation of boundary surfaces in tetrahedral meshes. The proposed solutions to boundary surface preservation, however, are often too restrictive. Some systems reject any edge collapses in the neighborhood of boundaries, while others do not allow collapses between boundary and internal vertices. 


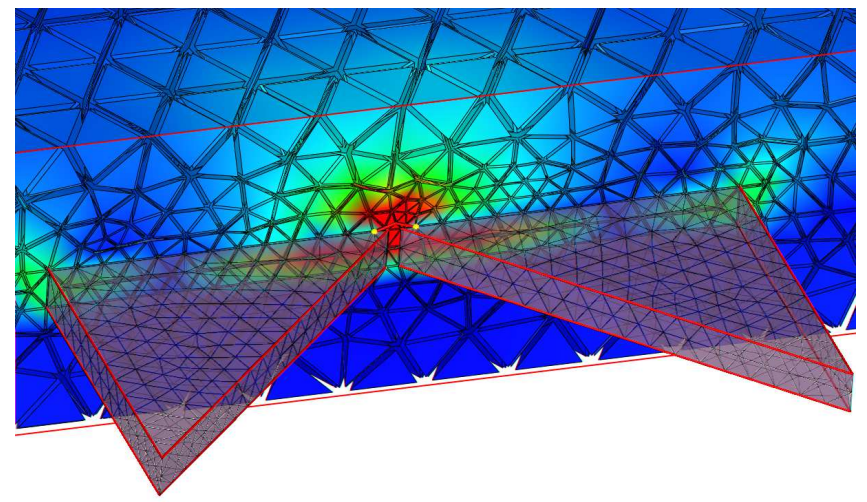

Fig. 1. Electromagnetism simulation for furtivity studies on a large irregular tetrahedral mesh where surface and linear substructures are the heart of the simulation as they influence the field propagation.

In contrast our system is less restrictive: edges may be collapsed anywhere in the mesh, even in the neighborhood of substructures, and even between a vertex in the substructures and a vertex outside the substructures. This leads to higher simplification rates. Furthermore the simplification is uniformly spread out across the mesh, regardless of the underlying substructures.

The main contribution of our paper is a robust algorithm to detect if an edge can be collapsed without modifying the topology of the mesh and of its substructures. Our system combines this topological validity test with simple geometric and numeric error measures in order to drive the simplification. However the focus of this paper is clearly on preserving topology - not on preserving the geometry of the mesh or the numerical values attached to it. As a matter of fact, the numerous geometric error measures proposed in other papers can be straightforwardly extended to take into account substructures in a mesh, whereas the preservation of the topology of the substructures is the real challenge.

In a previous work [14] we introduced a topological test for preserving polylines in non-manifold triangular meshes. Our current work extends the algorithms of [14] to tetrahedral meshes with 2D and 1D substructures. This extension is not trivial, both from a theoretical point of view and for the implementation. The latter is done very carefully in our system. Efficient data structures and algorithms are precisely described. Following [14], the mesh is modeled as a simplicial complex extended by new simplices connecting the substructures to a dummy vertex. The validity of the edge collapse is checked in the extended complex using results from [4]. Thus there is only one consistent test to ensure the preservation of the topology of the mesh and of all embedded substructures. In particular we do not rely on heuristic solutions that would treat the mesh and the substructures independently.

The remainder of the paper is structured as follows. Section 1.1 and 1.2 give some additional motivation for this work. Section 2 reviews related work. Section 3 presents the theory and Section 4 describes the implementation in detail. Section 5 presents 

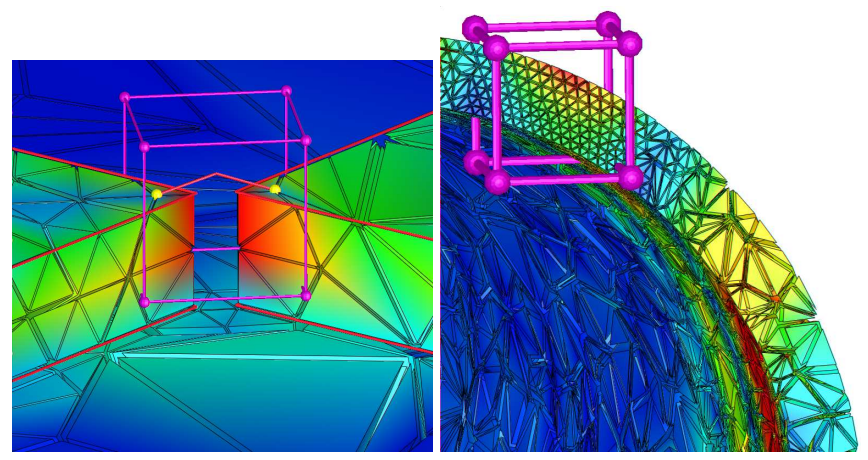

Fig. 2. (left) Detail of a substructure shown at high resolution only inside a volume of interest. The topology of the mesh and the substructures is preserved everywhere. (right) Multiresolution visualization of a thin layer of material with the associated electromagnetic field magnitude.

the integration of the topological test in our visualization system, with examples of application on real world datasets.

\subsection{Strength of the Topological Criteria}

Geometric error metrics in simplification are coupled with a threshold value that one can adapt to coarsen or refine the data. No matter how good the error computation is, the decision of removal greatly depends on this threshold which is application depend in order to handle unexpected cases often met in Scientific Visualization. In contrast, topological tools guaranty the integrity of the data after simplification without integrating global information or any metric between elements. Combinatorial Topology criteria are general to any application domain that uses meshes.

\subsection{Electromagnetic Simulation}

Electromagnetism is a wide field used in many applications such as electromagnetic compatibility, furtivity, or the modeling of new absorbing media. Stealth technology relies on the conception and simulation of new absorbing materials in order to decrease the signals reflected from the target to the radar receiver. This ability to reflect radar signals is characterized through Radar Cross Section (RCS) represented as a single scalar. However, to minimize this value, designers need to fully understand the electromagnetic field behavior on the object surface and in its interior. Numerical simulations of this phenomenon often use volumetric finite element methods coupled with a domain decomposition.

The main challenges when simplifying these data for multiresolution visualization come from the complex topology of the embedded structures and the large amount of data. Material boundaries (e.g. interfaces) and various substructures of different dimensions (possibly point sources and polylines) that exist in specific layers introduce a complex topology. The multiple crossing interfaces can be represented as one nonmanifold surface embedded in the volume mesh. The union of all polylines can also be 
seen as a unique linear structure which intersects the non-manifold surface. Figures 1 and 3 illustrate such data.

Also, due to the high frequency of the incident electromagnetic field, the mesh needs to be subdivided to the scale of the wave length leading to millions of cells. Because of the thin domain decomposition required by the numerical simulation and the design of specific objects, current tetrahedral meshes have only a few tetrahedra in the thickness of a layer (see Figure 7) even though the entire mesh may be composed of a hundred of millions of cells.

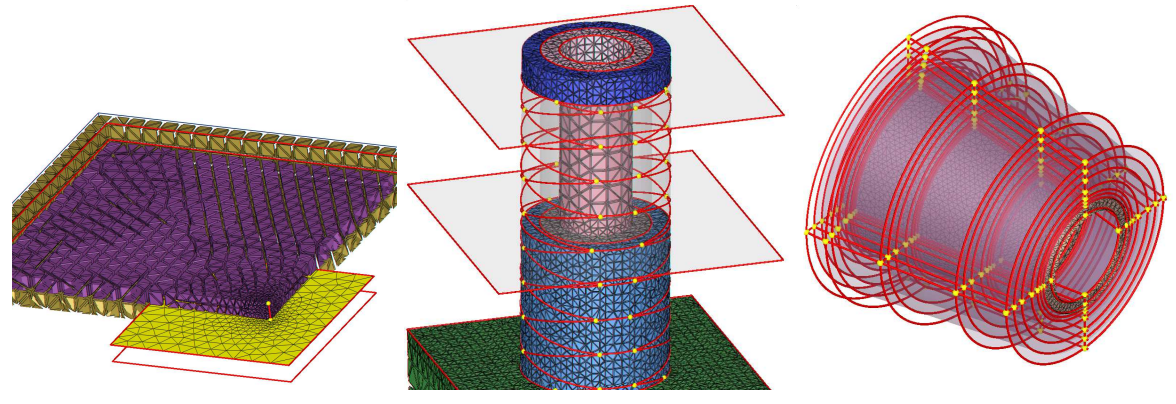

Fig. 3. (left) Polyline emerging from an orthogonal base plan. The mesh is subdivided around this structure. (center) Linear structure with multiple self intersections twisted around a cylindrical mass with orthogonal interfaces. (right) Multiple crossing interfaces of thin material layers and polylines of the structure.

Although we introduced our method on electromagnetism simulations, the exact same topological test can be apply to any domain where the topology of embedded structures need to be preserved trough simplification of the tetrahedral mesh (geology, medical imaging ...

\section{Related Work}

Multiresolution approaches provide techniques to efficiently and accurately explore large unstructured polygonal and volume data in Computer Graphics and Scientific Visualization. In order to create a multiresolution representation, many mesh simplification algorithms have been proposed over the last decade ranging from surface to volume based methods. As we focus on topology criteria, we will only survey these criteria used in previous algorithm and dismissed geometry/attribute error metrics and surface/volume mesh simplification algorithm [10,2]. In fact any error metrics can be combined to our algorithm without any side effect in order to improve the attribute or geometric quality of the simplified mesh.

Among the wide range of simplification methods, only a few of them introduce specific topological tests and almost none of them tackle the problem of linear/surface features in a volume mesh. Early work on topological criteria has been done on sur- 
face simplification in [13] where the vertices candidate to the removal are classified by analyzing their local neighborhood.

With notions taken from the computational topology, [8] has proven that the topology of a triangular mesh can be preserved, through simplification, if the 3 tests introduced in the paper are fulfilled. Later, in the PM method [7], these tests are completed with local heuristics in order to preserve the topology of discontinuity curves defined on a surface. The author proposes 6 rules based on an enumeration of the vertices around an edge representing a linear feature.

[9] successfully simplify non-convex tetrahedral meshes by triangulating the non convex regions. The authors check for tetrahedron flipping avoiding topological and geometrical problems only at the boundary. However, the authors point out that some topological problems can still be undetected with their test during the simplification. All these previous topological criteria are not general enough to preserve the topology of both 1D and 2D structures in a 3D mesh.

[4] is the groundbreaking paper on topology preservation. It has introduced the link conditions to robustly check if an edge collapse preserves the topology of a 3-complex. Our paper makes extensive use of these link conditions (reviewed in Section 3).

Intuitively, the link of a simplex $u$ is the subset of simplicies being in contact with all adjacent simplicies of $u$ and the rest of the mesh. In a 3-complex without boundary, [4] has proven that an edge collapse $u v$ preserves the topology of the complex if the intersection of the vertex links is equal to the edge link. This condition is known as the link condition. For general 3-complexes, this link condition has to be evaluated in a succession of complexes built by iteratively filtering the simplicies having a simpler topological neighborhood.

Extending the multiresolution representation of [3], [1] first integrates the link conditions to preserve the topology of both the interior cells and the boundary of the mesh. [12] also uses the link conditions but simplifies them by assuming that the tetrahedral mesh is manifold. The link condition is computed for a 3-complex without boundary for the interior tetrahedra and if a boundary tetrahedron is encountered a second link condition for the 2-complex at the boundary is added.

Because none of these papers fullfil our needs we introduced a new algorithm which successfuly simplifies general simplifical 3-complex in preserving both the topology of this complex anf the topology of sub-complexes embedded into it. The strength of this new algorithm comes from: $(i)$ its locality, (It only uses the direct neighborhood of the simplicies) (ii) its unicity, (It can handle any complicated topological neighborhood regardless of its dimension lower than 3.) and (iii) its exactness (It is based on theorical results taken from combinatorial topology rather than local heuristics).

\section{Topology preserving edge collapse in an extended 3-complex}

\subsection{Link conditions in a 3-complex}

The link conditions introduced in [4] can be used in a tetrahedral mesh to test if an edge $e$ between two vertices $u$ and $v$ can be collapsed without modifying the topology of the mesh. The mesh is modeled as a simplicial complex $K$. The link conditions are 
expressed using basic operators from algebraic topology. We assume the reader is familiar with elementary notions in this field. Precise definitions of the link Lk $T$, the star St $T$ and the closure $\bar{T}$ of a set $T$ of simplices can be found in [11] and illustrated in [14].

If $K$ is a manifold 3-complex, the link conditions reduces to:

$$
\text { Lk } u \cap \operatorname{Lk} v=\mathrm{Lk} e
$$

If the 3-complex is non-manifold, the condition (1) has to be tested in three successive smaller complexes of dimensions 3,2 and 1. This is detailed in Section 3.3.

\subsection{Extending the mesh with the substructures}

In order to use this link condition to our setting, the embedded structure need to be visible from the surrounding mesh. To do so, we increase the neighborhood complexity of simplicies defining the sub-structures as follow. The tetrahedral mesh is modeled as a simplicial complex $K$. The 2D (resp. 1D) substructures are defined as a set $L_{f}$ (resp. $L_{e}$ ) of faces (resp. edges) in $K$. We define an extended complex $\widetilde{K}$ by adding to $K$ the cones from a dummy vertex $\omega$ to each simplex in the closure of the substructures, as illustrated in Figure 4. The key idea is to consistently encode the topology of the mesh

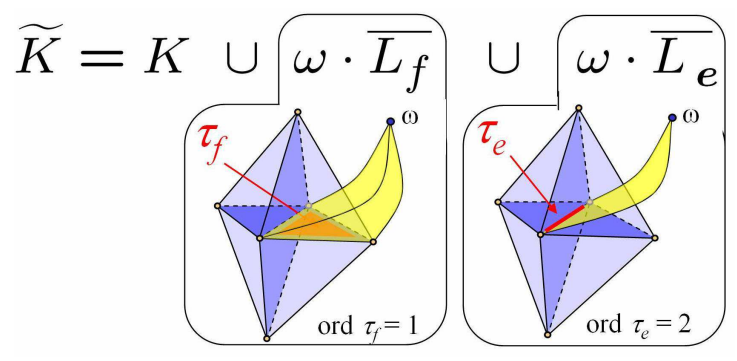

Fig. 4. Construction of the extended complex. The red simplicies correspond to the substructures $L_{f}$ and $L_{e}$. The yellow simplicies represent the elements added to the original complex $K$ (shown in blue) in order to extend $K$ to $\widetilde{K}$.

and its substructures in a single extended complex.

\subsection{Link conditions in the extended complex}

To preserve the topology of the mesh and its substructures, we apply the link conditions in the extended complex defined in Section 3.2. Even if the original mesh is manifold, the extended complex is non manifold. Thus, the link condition (1) must be evaluated respectively in the following complexes:

$$
\begin{aligned}
\widetilde{K}^{\omega} & =\widetilde{K} \cup\left(\omega \cdot \mathrm{Bd}_{1} \widetilde{K}\right) \\
\widetilde{G}^{\omega} & =\mathrm{Bd}_{1} \widetilde{K} \cup\left(\omega \cdot \mathrm{Bd}_{2} \widetilde{K}\right) \\
\widetilde{H}^{\omega} & =\mathrm{Bd}_{2} \widetilde{K} \cup\left(\omega \cdot \mathrm{Bd}_{3} \widetilde{K}\right)
\end{aligned}
$$


where $\mathrm{Bd}_{i} \widetilde{K}$ is the set of all simplicies of order $i$ or less in $\widetilde{K}$. The link of a simplex in $\widetilde{K}^{\omega}, \widetilde{G}^{\omega}$ and $\widetilde{H}^{\omega}$ is respectively noted as $\mathrm{Lk}_{0}^{\omega}, \mathrm{Lk}_{1}^{\omega}$ and $\mathrm{Lk}_{2}^{\omega}$. The precise definition of the order of a simplex is given in [4]. Simplicies of dimension $k$ in a 3-complex have an order not greater than $3-k$. Therefore $\widetilde{K}^{\omega}$ is a 3 -complex, while $\widetilde{G}^{\omega}$ contains only edges and $\widetilde{H}^{\omega}$ only vertices. Note that our algorithm can deal with non-manifold tetrahedral meshes. However for clarity reasons, the following discussion is restricted to the case where the tetrahedral mesh is a manifold with boundary.

In this case, $\mathrm{Bd}_{1} \widetilde{K}$ contains the boundary faces of $K$ and the faces of $L_{f}$. It contains also the edges and vertices of these faces. $\mathrm{Bd}_{2} \widetilde{K}$ contains the edges of $L_{e}$ and the edges adjacent to exactly one, or else three or more faces of $L_{f}$ (i.e., the boundary and the non-manifold edges of $L_{f}$ ). It contains also the vertices of these edges. Finally $\mathrm{Bd}_{3} \widetilde{K}$ contains the vertices adjacent to one or else three or more edges of $\mathrm{Bd}_{2} \widetilde{K}$ (i.e. the boundary and non-manifold vertices of $\mathrm{Bd}_{2} \widetilde{K}$ ). This includes vertices at the intersections created when a polyline in $L_{e}$ crosses a surface in $L_{f}$.

By experience, the simplicies of high order correspond to regions of interest that the user visualizes in detail. Important features in the mesh (detected by cells marked as simplices of high order) are usually correlated with high variations in the data. This point is clearly illustrated in the electromagnetic simulations where the field greatly varies around the linear structure or the points of contact in between this element and a mass plan.

\section{Implementation of the Topology preservation test}

\subsection{Datastructure of the embedded simplices}

The implementation of the link condition requires to iterate through all adjacent simplicies around a vertex and an edge. For memory requirement and efficiency we do not store explicitly all simplicies (faces, edges and vertices) of our substructures. Instead, as both the substructures and the link simplicies are embedded in cells of the original tetrahedral mesh, all of them are encoded as relative element indices in a tetrahedron. For each tetrahedron 4 bits (resp. 6 bits) are used to indicate which faces (resp. edges) support a 2D (resp 1D) substructure. Both the triangle mesh connectivity of the 2D substructures and the linear mesh connectivity of the 1D substructures are retrieved through the tetrahedron mesh connectivity without using additional memory. Moreover, during the simplification process, such a datastructure eases and optimizes the updates of the substructures after an edge collapse. A modification on the tetrahedral mesh will be directly passed on to the substructures, without any additionnal treatment.

\subsection{Algorithmic implementation}

The algorithm 1 describes the evaluation of the link conditions in order to detect if the collapse of the edge $e=u v$ preserves both the topology of the mesh and the topology of any embedded structure. The extraction of the link of a simplex is the most repeated operation when evaluating the link conditions. It is also time consuming as the star of the reference simplex has to be exhaustively visited. 


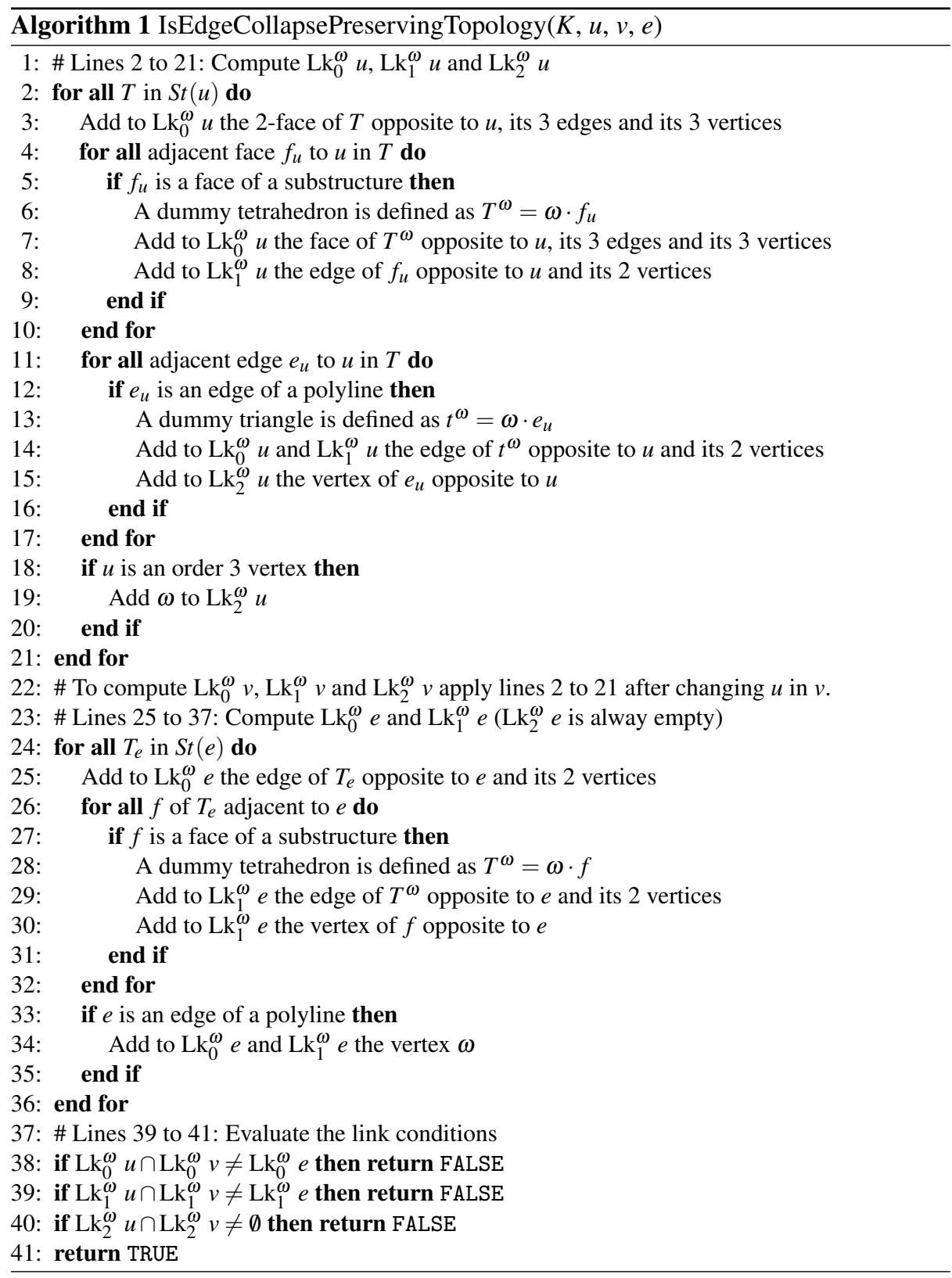



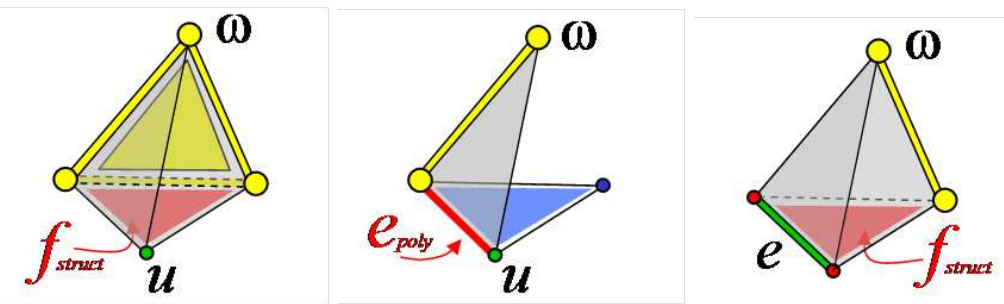

Fig. 5. Contribution to the links from the extended simplicies around a reference simplex $(u$ or $e$ shown in green). $\omega$ represents the dummy vertex. The substructures are shown in red and the simplicies to insert into the links in yellow.

The links are directly obtained without explicitly building the extended complex.

In order to understand this optimized implementation, one has to associate the different members of equation (2) to the algorithm steps. For example, starting at line 5 and illustrated on figure 5 le ft, if $f_{u}$ is a face of a substructure then the dummy tetrahedron $T^{\omega}$ increases the order of $f_{u}$ to 1 . Thus, $f_{u}$ is included in $\mathrm{Bd}_{1} \widetilde{K}$ and contributes to $\mathrm{Lk}_{0}^{\omega}$ but also to $\mathrm{Lk}_{1}^{\omega}$ because of the member $\omega \cdot \mathrm{Bd}_{1} \widetilde{K}$ of $\widetilde{G}^{\omega}$ (line 8 ). The simplices represented in yellow on the figure 5 middle are the contribution of the triangle $t^{\omega}$ defined on line 13 to $\mathrm{Lk}_{0}^{\omega}$ and $\mathrm{Lk}_{1}^{\omega}$. This extension increases the order of the edge $e_{u}$ to 2 making it visible in $\mathrm{Bd}_{2} \widetilde{K}$. Thus, line 15 of the algorithm implements the contribution of the simplicies present in the member $\mathrm{Bd}_{2} \widetilde{K}$ of $\widetilde{H}^{\omega}$. A last example, shown on figure 5 right, illustrates the contribution of the dummy tetrahedron $T^{\omega}$ introduced on line 29. In this case only the opposite edge (yellow) to $e$ (green) is inserted to the link of $\mathrm{Lk}_{0}^{\omega} e$ as no face can be present in the link of an edge.

One has to note that due to the strong theoretical background, the topological criteria is insensitive to the complexity of the neighborhood of the collapse (multiple surfaces and polylines).

\section{Applications}

\subsection{Volumetric Simplification}

This section presents various applications of the topology preserving visualization system introduced in the present paper.

The example on figure 6 shows the simplification of a mesh used in electromagnetism simulation. The object is composed of approximatively twenty thin layer of materials encapsulated in each others. The geometry is meshed with about 2 millions of tetrahedra. In order to study the circulation of the electromagnetic field at the boundary of each materials, all interfaces are explicitely described as embedded surfaces. Additional linear structures with self intersections are inserted in the model. Figure 6(b) shows the simplified mesh after removing $90 \%$ of the vertices with edge collapses. This example illustrates the strength of the algorithm on data composed of a large amount of 


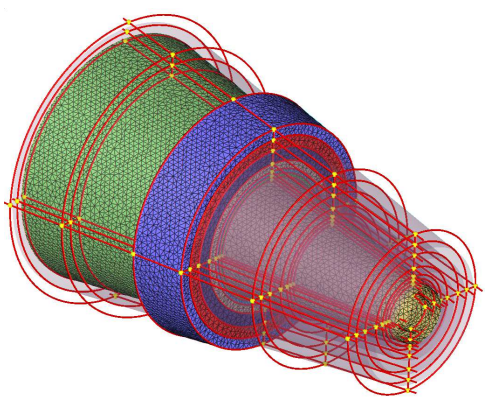

(a) Original mesh

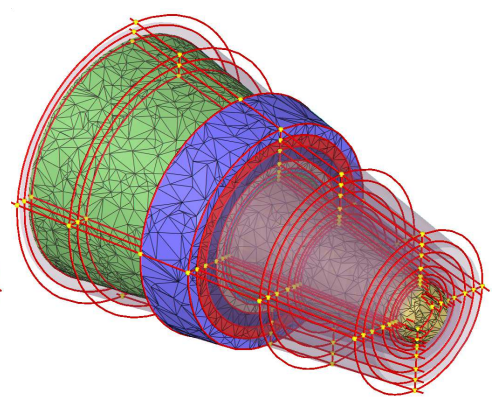

(b) After $90 \%$ of simplification

Fig. 6. Volumetric simplification of a model with multiple substructures. A few material layers are shown as opaque triangle mesh and all interfaces as semi-transparent surfaces. The linear features are in red and their self intersections marked by yellow shperes.

substructures of different dimension with multiple self intersections (e.g. increasing the topological complexity).

Achieving aggressive simplification rates becomes challenging when dealing with large volumetric mesh organised in thin material layers. Figure 7 shows a detail of such

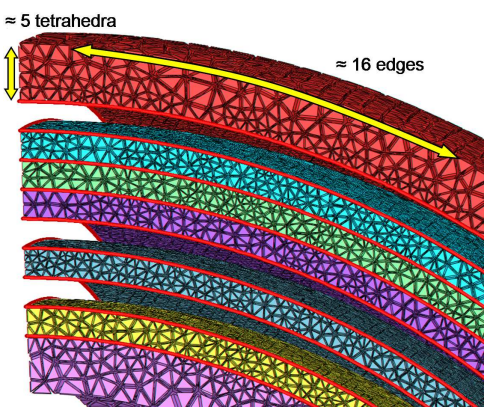

(a) Original mesh

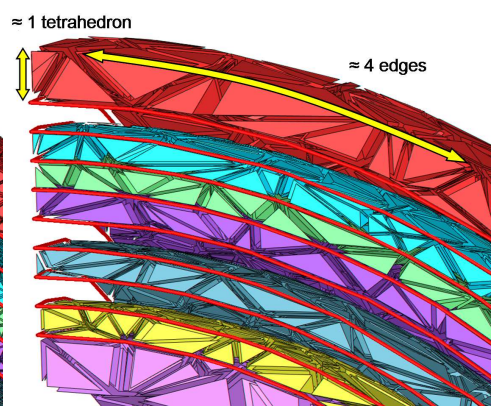

(b) After $98 \%$ of simplification

Fig. 7. Aggressive simplification of a volume mesh composed of thin material layers. The topological criteria preserve both the thickness of the layers and the topology of the substructures while allowing collapses of edges on the substructures.

data where the material layers have no more than 5 tetrahedra in their thickness. Linear features are also defined on specific boundaries. Figure 7(b) shows the result after a massive simplification where $98 \%$ of the vertices have been removed by edge collapses. In this example the simplification stopped when all candidate edges are rejected due to the topological criteria. It is important to note that the topological constraints on the 2D interfaces insure the preservation of the volume of each layer (at least 1 tetrahedron in the thickness and usually no more). Another interesting aspect of this example shows 
that strong constraints on the substructure topology do not disturb or prevent an high simplification rate of the substructures. The arrow drawn along a linear feature living on an interface covers about 16 edges on the initial mesh but only 4 edges after simplification. Thus many edges of the interface and of the polyline have been collapsed.

\subsection{Multiresolution Visualization}
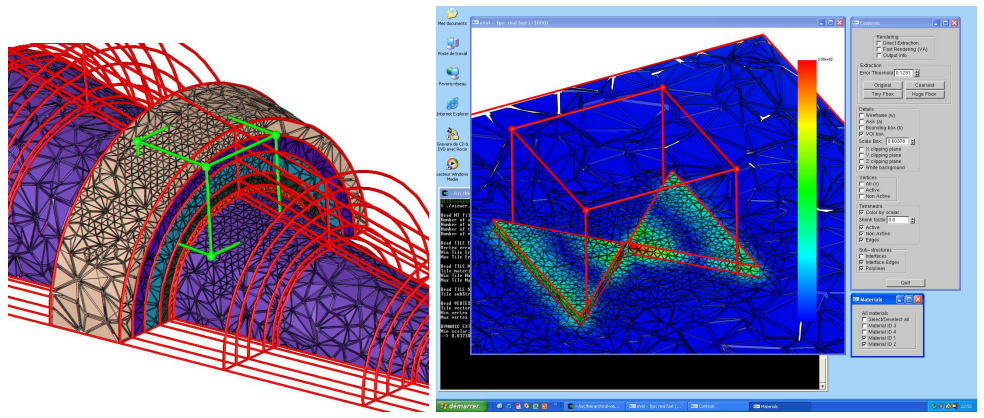

Fig. 8. $(l e f t)$ Variable resolution visualization of a volume mesh with multiple linear features. The topology of the substructures is guaranteed to be preserved. (right) Snapshot of the multiresolution visualization tool to explore simulation data with embedded structures on a desktop PC.

In order to provide a visualization system taking advantage of the substructure topology preserving criteria, the algorithm has been integrated to an existing multiresolution framework. The MT library [5], freely available and based on the formal approach of [6], provides the tools to build and exploit a multiresolution representation from a sequence of valid transformation on a mesh. MT stores a partial order amongst the modifications into a DAG which can then be querried with static or dynamic criteria to produce various resolutions. Global or local extractors allow the user to extract meshes at variable resolution centered around a volume of interest (VOI). Figures 8 and 1 show a multiresolution mesh at high resolution only inside a VOI interactively moved by a user. The mesh at low resolution reduces the use of the graphic processor allowing the application to maintain an interactive frame rate even with an original mesh of several millions of cells. As the substructure preserving topology criteria have been used to build this representation, the topology of the substructures is guaranteed to be preserved on the multiresolution visualizations.

\section{Conclusion}

This paper has introduced new results for simplifying tetrahedral meshes with $2 \mathrm{D}$ and $1 \mathrm{D}$ substructures. As a specific application our system can simplify multi-material tetrahedral meshes while accurately preserving the topology of the material parts. To the 
best of our knowledge, this is the first paper that deals with the simplification of multimaterial tetrahedral meshes. Our system can also handle more general 2D and 1D substructures. An application in the field of electromagnetism simulation, from which the system originates, has been shown. This application and the technology explained (multiresolution) are part of the strategy of the CEA in order to visualize large data computed on a remote super-computer. While focusing on topology preservation, our system can accommodate any geometric and numeric error measures as well. Once the simplification is computed, the system uses the MT library to visualize the mesh with higher precision inside a VOI. In future work we plan to optimize the VOI extraction by developing algorithms specifically designed for handling tetrahedral meshes with substructures as well as better attribute and geometric attribute metrics to drive the simplification.

\section{References}

1. P. Cignoni, D. Costanza, C. Montani, C. Rocchini, and R.Scopigno. Simplification of tetrahedral volume with accurate error evaluation. In Proceedings IEEE Visualization '00, pages 85-92, 2000.

2. P. Cignoni, L. D. Floriani, P. Lindstrom, V. Pascucci, J. Rossignac, and C. Silva. Multiresolution modeling, visualization and streaming of volume meshes. In Eurographics 2004, Tutorials 2: Multi-resolution Modeling, Visualization and Streaming of Volume Meshes. INRIA and the Eurographics Association, September 2004.

3. P. Cignoni, C. Montani, E. Puppo, and R. Scopigno. Multiresolution representation and visualization of volume data. IEEE Transactions on Visualization and Computer Graphics, 3(4):352-369, oct-dec 1997.

4. T. Dey, H. Edelsbrunner, S. Guha, and D. Nekhayev. Topology preserving edge contraction. Publications de l'Institut de Mathématiques (BEOGRAD), 66(80):23-45, 1999.

5. L. D. Floriani, P. Magillo, and E. Puppo. The MT (Multi-Tesselation) package. DISI, University of Genova, Italy, http://www.disi.unige.it/person/MagilloP/MT, Jan. 2000.

6. L. D. Floriani, E. Puppo, and P. Magillo. A formal approach to multiresolution modeling. In W. Straer, R. Klein, and R. Rau, editors, Theory and Practice of Geometric Modeling. SpringerVerlag, 1997., 1997.

7. H. Hoppe. Progressive meshes. Computer Graphics, 30(Annual Conference Series):99-108, 1996.

8. H. Hoppe, T. DeRose, T. Duchamp, J. McDonald, and W. Stuetzle. Mesh optimization. Technical Report TR 93-01-01, Dept. of Computer Science and Engineering, University of Washington, Jan. 1993.

9. M. Kraus and T. Ertl. Simplification of nonconvex tetrahedral meshes, 2000.

10. D. Luebke. A developer's survey of polygonal simplification algorithms. IEEE Computer Graphics and Applications, 21(3):24-35, 2001.

11. J. Munkres. Elements of algebraic topology. Perseus publishing, Cambridge, 1984.

12. V. Natarajan and H. Edelsbrunner. Simplification of three-dimensional density maps. IEEE Transactions on Visualization and Computer Graphics, 10(5):587-597, 2004.

13. W. Schroeder, J. Zarge, and W. Lorensen. Decimation of triangle meshes. Computer Graphics, 26(2):65-70, 1992.

14. F. Vivodtzev, G.-P. Bonneau, and P. Letexier. Topology preserving simplification of $2 \mathrm{~d}$ nonmanifold meshes with embedded structures. The Visual Computer, 21(8):679-688, 2005. 